\title{
Involvement of PBRM1 in VHL disease-associated clear cell renal cell carcinoma and its putative relationship with the HIF pathway
}

\author{
SOPHIE GAD ${ }^{1,2}$, GWENAËL LE TEUFF ${ }^{3,4}$, BAPTISTE NGUYEN ${ }^{1}$, VIRGINIE VERKARRE ${ }^{5-7}$, \\ VERONIQUE DUCHATELLE ${ }^{8}$, VINCENT MOLINIE $^{8,9}$, KATIA POSSEME $^{10}$, BENJAMIN GRANDON $^{1}$, \\ MELANIE DA COSTA $^{1}$, BASTIEN JOB ${ }^{11}$, GUILLAUME MEURICE ${ }^{11}$, NATHALIE DROIN ${ }^{12}$, \\ ARNAUD MEJEAN $^{7,13}$, SOPHIE COUVE ${ }^{1,2}$, FLORE RENAUD ${ }^{1,2}$, BETTY GARDIE $^{1,14}$, \\ BIN TEAN TEH ${ }^{15,16}$, STEPHANE RICHARD ${ }^{1,2,7}$ and SOPHIE FERLICOT ${ }^{2,7,10}$
}

${ }^{1}$ Ecole Pratique des Hautes Etudes (EPHE), Paris Sciences Lettres Research University, 75014 Paris;

${ }^{2}$ Mixed Research Unit (UMR) 9019, Gustave Roussy Institute, French National Scientific Research Center (CNRS),

Paris-Saclay University; ${ }^{3}$ Department of Biostatistics and Epidemiology, Gustave Roussy Institute, CNRS,

Paris-Saclay University; ${ }^{4}$ French National Health and Medical Research Institute (INSERM),

Research Center in Epidemiology and Population Health (CESP), Paris-Saclay School of Medicine, Paris-Saclay University, 94800 Villejuif; ${ }^{5}$ Department of Pathology, Public Hospitals of Paris (AP-HP) Centre, Georges Pompidou European Hospital, Paris University; ${ }^{6}$ INSERM UMR 970, Paris Cardiovascular Research Center (PARCC), Georges Pompidou European Hospital,

75015 Paris; ${ }^{7}$ Department of Urology, PREDIR French National Cancer Institute (INCa), AP-HP, Bicêtre Hospital,

94270 Le Kremlin-Bicêtre; ${ }^{8}$ Department of Pathology, Saint-Joseph Hospital, 75014 Paris; ${ }^{9}$ Department of Pathology,

Aix-en-Provence Hospital Center, 13616 Aix en Provence; ${ }^{10}$ Department of Pathology, AP-HP, Bicêtre Hospital,

Paris-Saclay University, 94270 Le Kremlin-Bicêtre; ${ }^{11}$ Bioinformatics Core Facility, Gustave Roussy Institute, CNRS, Paris-Saclay University; ${ }^{12}$ Genomics Core Facility, Gustave Roussy Institute, CNRS, Paris-Saclay University, 94800 Villejuif;

${ }^{13}$ Department of Urology, AP-HP, Georges Pompidou European Hospital, Paris University, 75015 Paris;

${ }^{14}$ L'Institut du Thorax, INSERM, CNRS, Nantes University, 44000 Nantes, France; ${ }^{15}$ Program in Cancer and Stem Cell Biology, Duke-National University of Singapore (NUS) Medical School, Singapore 169610; ${ }^{16}$ Laboratory of Cancer

Epigenome, Division of Medical Science, National Cancer Centre, Singapore 169610, Republic of Singapore

Received May 25, 2021; Accepted August 3, 2021

DOI: 10.3892/ol.2021.13096

\begin{abstract}
Von Hippel-Lindau (VHL) disease is the main cause of inherited clear-cell renal cell carcinoma (ccRCC) and is caused by germline mutations in the $V H L$ tumor suppressor gene. Bi-allelic VHL alterations lead to inactivation of $\mathrm{pVHL}$,
\end{abstract}

Correspondence to: Dr Sophie Ferlicot, Department of Pathology, AP-HP, Bicêtre Hospital, Paris-Saclay University, 78 Général Leclerc Street, 94270 Le Kremlin-Bicêtre, France

E-mail: sophie.ferlicot@aphp.fr

Abbreviations: CGH, comparative genomic hybridization; ccRCC, clear-cell renal cell carcinoma; HIF, hypoxia inducible factor; IHC, immunohistochemistry; ISUP, International Society of Urological Pathology; PBRM1, polybromo 1; VHL, Von Hippel-Lindau; wt, wild-type

Key words: clear cell renal cell carcinoma, von Hippel-Lindau disease, Von Hippel-Lindau germline mutation, polybromo 1 somatic mutation, BAF180, hypoxia inducible factor 1 , hypoxia inducible factor 2 which plays a major role by downstream activation of the hypoxia inducible factor (HIF) pathway. Somatic VHL mutations occur in $80 \%$ of sporadic ccRCC cases and the second most frequently mutated gene is polybromo 1 (PBRM1). As there is currently no data regarding $P B R M 1$ involvement in VHL disease-associated ccRCC, the aim of the present study was to assess the PBRM1 mutational status, and PBRM1 and HIF expression in VHL disease-associated ccRCC series compared with a sporadic series. PBRMI gene was screened by Sanger sequencing for 23 VHL-disease-associated ccRCC and 22 sporadic ccRCC cases. Immunohistochemical studies were performed to detect the expression of PBRM1, HIF1 and HIF2 for all cases. In VHL-associated tumors, $13.0 \%(n=3 / 23)$ had PBRM1 somatic mutations and $17.4 \%(\mathrm{n}=4 / 23)$ had a loss of PBRM1 nuclear expression. In sporadic cases, $27.3 \%(n=6 / 22)$ showed PBRM1 somatic mutations and $45.5 \%(\mathrm{n}=10 / 22)$ had a loss of PBRM1 nuclear expression. Loss of PBRM1 was associated with an advanced tumor stage. HIF1-positive tumors were observed more frequently in the VHL-associated ccRCC than in the sporadic series. Furthermore, in the VHL cohort, PBRM1 expression appeared to be associated more with HIF1 than with HIF2. Given that hereditary tumors tend to be less aggressive, these results would suggest that co-expression 
of PBRM1 and HIF1 may have a less oncogenic role in VHL-associated ccRCC.

\section{Introduction}

Kidney cancer accounts for $3 \%$ of all adult cancers worldwide and clear cell renal cell carcinoma (ccRCC) represents the main histological subtype (70\% of all renal cancers) $(1,2)$. Approximately 3\% of RCC are linked to an inherited predisposition. Von Hippel-Lindau (VHL) disease is the most frequent inherited RCC syndrome, with an incidence of $1 / 36,000$ births and a predisposition for the occurrence of ccRCC in up to $50 \%$ of VHL patients (3). Bi-allelic inactivation of the $V H L$ tumor suppressor gene, located at 3p25-26, is frequently observed in VHL disease-associated ccRCC. Furthermore, loss of $3 p$ and somatic mutations of $V H L$ occur in $90 \%$ and approximately $80 \%$ of sporadic ccRCC, respectively. Loss of $V H L$ function in ccRCC leads to stabilization of the hypoxia-inducible transcription factors HIF1alpha (HIF1) and HIF2alpha (HIF2), thereby inducing the expression of genes that regulate metabolism, angiogenesis, cell proliferation, and the epithelial-mesenchymal transition (4). Thus, a HIF-mediated transcription response is a key component of ccRCC pathogenesis. Gordan et al reported that ccRCC can be segregated into three groups according to the HIF staining defining: no HIF expression, $\mathrm{HIF} 2^{+}$, and $\mathrm{HIF} 1^{+} / \mathrm{HIF} 2^{+}$tumor subtypes (5). Both HIF1 and HIF2 are thought to have promoting roles in ccRCC carcinogenesis, although studies performed in animal and cell lines are contradictory, some suggesting that HIF2 would rather have a tumorigenic activity, whereas HIF1 tends to have a tumor-suppressive role (6-9). However, $V H L$ loss alone is not sufficient to cause ccRCC $(10,11)$. In sporadic ccRCC, recent studies using next-generation sequencing (NGS) have identified new frequently mutated genes located in the $3 p$ loss region near the VHL locus: PBRM1, BAPI, and SETD2 (12-15). With a somatic mutation rate of 29 to $40 \%$, PBRMI (PolyBromo-1) is the most frequently mutated gene after $V H L$ in ccRCC. Interestingly, it has also been reported to be a new predisposing gene for hereditary ccRCC (16). PBRM1 encodes BAF180 (hereafter referred to as PBRM1), which is a member of the human ATP-dependent chromatin remodeling Switch/Sucrose Non-Fermentable B (SWI/SNF) analog complex PBAF (17). Members of this family have a number of critical roles in many physiological cellular processes including DNA replication, recombination, and repair, as well as cell death, cell fate, and the initiation or progression of cancer (18). Moreover, a number of authors have recently studied the relationship between PBRMI and HIFs, although the results have been contradictory $(19,20)$. In one of these studies, inactivation of PBRMI increased the HIF response in VHL-deficient cell lines irrespective of HIF1 and HIF2 (19). In the second study, the role of $P B R M 1$ was dependent on the HIF subtypes of the tumors. PBRM1 expression was pro-oncogenic in $\mathrm{HIF}^{+}$tumors and tumor-suppressive in $\mathrm{HIF}^{+} / \mathrm{HIF}^{+}$tumors (20). These initial results in sporadic ccRCC highlight the need to further investigate the link between PBRM1 and HIF expression in VHL ccRCC. To the best of our knowledge, no study has focused on this link in a cohort of renal cell carcinomas from VHL patients. It seems important to study this cohort given that hereditary cancers represent a model of carcinogenesis for better understanding the sporadic ccRCC. It has been shown that VHL disease-associated tumors have genomic profiles that are similar to that of a subgroup of sporadic ccRCC (21). However, due to the early detection of ccRCC in VHL patients, the frequency of PBRM1 somatic mutations needs to be evaluated in order to determine whether VHL-associated ccRCC and sporadic ccRCC follow distinct pathways or whether the same model of carcinogenesis applies (22). In order to investigate this, we firstly used sequencing analysis and immunohistochemistry (IHC) to evaluate the PBRM1 mutational status and PBRM1 expression in a VHL-associated ccRCC series vs. a sporadic ccRCC series. Secondly, we used IHC to compare the HIF pattern of staining with PBRM1 expression in both of the RCC series.

\section{Materials and methods}

Patient characteristics and tumor tissue samples. Tumor tissue samples from a series of $45 \mathrm{ccRCC}$ patients were obtained courtesy of the French NCI network on inherited RCC coordinated by Prof. S. Richard (PREDIR, Bicêtre Hospital, France). The primary tumor, and for some cases also adjacent non-tumor samples, were obtained from these patients who had undergone surgical tumor resection. All of the patients had provided written informed consent before surgery for use of their tumors. The tumor samples were either immediately frozen in liquid nitrogen or fixed in formalin after the surgery. A representative block was selected for immunohistochemistry from all haematoxylin and eosin-stained sections. Pathological data (tumor stage and ISUP nucleolar grade) were reevaluated by the same uropathologist (SF) according to the most recent ISUP/World Health Organization/AJCC classification (23). The stage and the nucleolar grade were dichotomized in both categories: low (T1-T2, G1-G2) and high (T3-T4, G3-G4). Part of the series was previously described $(24,25)$.

DNA extraction and PBRMI sequencing. Tumor DNA was extracted using a Qiagen DNeasy ${ }^{\circledR}$ Blood and Tissue kit. The DNA concentration and purity were determined by using a Qubit Fluorometer (Invitrogen). The mutational status of PBRM1 was determined by direct sequencing of this gene as previously described (16). Briefly, all 30 exons and exon-intron junctions were screened by the Sanger method for the 45 tumors. The mutations were annotated according to gene accession number ENST00000296302 (Table SI).

CGH array hybridization and data analysis. The DNA was labeled according to the Agilent Technologies protocol. For each tumor sample and gender-matched pooled normal control DNA from Promega (Human Genomic DNA), 500 ng of DNA was fragmented by heating. The tumor DNA and the control DNA were enzymatically labeled using an Agilent Genomic DNA Labeling Kit PLUS (Agilent, 5188-5309). The tumor samples were labeled with ULS-Cy5 and the control DNA with ULS-Cy3, and then hybridized to $244 \mathrm{~K}$ Human Whole-Genome arrays (G441B) from Agilent at $65^{\circ} \mathrm{C}$ for $48 \mathrm{~h}$ in a rotating chamber at $20 \mathrm{rpm}$.

After washing, the slides were scanned with an Agilent G2505B DNA Microarray Scanner at a resolution of $5 \mu \mathrm{m}$, 
using default parameters. The acquisition of signals from the resulting scanned images and normalization were performed with Feature Extraction v9.5 software (Agilent Technologies), using default parameters. The normalized data were recentralized using a custom in-house script and then analyzed with CGH Analytics v3.4.40 software, using the ADM-2 segmentation method with a threshold setting of 6 . The probes were mapped on the human genome build 36 (UCSC hg18, March 2006).

Immunohistochemical staining. Immunohistochemical staining was performed on $4-\mu \mathrm{m}$ thick paraffin whole-tissue sections on a semi-automated Bond Leica instrument. The primary antibodies that were used were: rabbit polyclonal anti-PBRM1 antibody (Bethyl Laboratories); mouse monoclonal anti-HIF1alpha antibody (clone MGC3; Abcam); and mouse monoclonal anti-HIF2alpha (clone ep190b; Abcam). The sections were treated with a solution of peroxidase-labeled streptavidin and the color reaction was developed by incubation with 3,3'-diaminobenzidine (DAB) according to the Bond Polymer Refine detection kit instructions. Nuclear counterstaining with hematoxylin was then performed.

The positive and negative controls yielded appropriate results for each procedure. Lymphocytes, stromal fibroblasts, and endothelial cells served as internal positive controls for the nuclear staining of PBRM1. Placenta tissue and colon adenocarcinoma samples were used as positive controls for nuclear staining of HIF1alpha and HIF2alpha, respectively. A negative staining control was performed without a primary antibody for each staining procedure and these sections exhibited no specific immunoreactivity. The staining was scored by a single uropathologist (SF) blinded to the $V H L$ and the PBRMI gene status.

PBRM1 expression was evaluated based on the percentage of PBRM1-positive cells. As described in a previous study evaluating PBRM1 expression as a continuous variable, we applied a cut-off value of more than $50 \%$ positive tumor cells as being indicative of a PBRM1-positive tumor (26).

For the HIF staining, a conventional cut-off of more than $5 \%$ positive cells was used to prevent false positivity. The tumors were stratified into four subtypes: HIF1 ${ }^{+}, \mathrm{HIF}^{+}$, $\mathrm{HIF}^{+} / \mathrm{HIF}^{+}$, and double negative. Thus, the results of the IHC staining were considered as a binary variable (negative or positive) for the statistical analyses.

Statistical analysis. The clinico-pathological characteristics of the VHL and the sporadic ccRCC were compared using the $\mathrm{Chi}^{2}$ or Fisher's exact tests for the qualitative variables and the Kruskal-Wallis test for the quantitative variables. The Cochran-Mantel-Haenszel test was used to study the association between two categorical variables controlling for a variable. The statistical analyses were performed using SAS software version 9.4 .

\section{Results}

Patient and tumor characteristics. We initially selected a series of 23 patients affected with von Hippel-Lindau disease who had been diagnosed with ccRCC (16 men and 7 women). In order to compare clinico-pathological and genetic characteristics, a prospective series of 22 patients with sporadic ccRCC (12 men and 10 women) was used. The clinico-pathological and genetic characteristics are detailed in Table I. No significant differences were found in terms of the sex-ratio between both series (VHL vs. sporadic cases) $(\mathrm{P}=0.30)$ although significant differences were observed for the age at the time of the diagnosis, with earlier diagnoses in VHL (median age of 40 years) compared to the sporadic group (median age of 69 years) (Chi-Square $\mathrm{P}<0.0001$ ). Regarding the stage and the ISUP grade, there were no significant differences between both ccRCC series $(\mathrm{P}=0.18$ and 0.88 respectively). As expected, the VHL-ccRCC all had mutated VHL (100\%), while the sporadic ccRCC had a lower rate of VHL mutation $(72.7 \%)(\mathrm{P}=0.01)$.

PBRM1 mutational status and protein staining. Direct sequencing of $P B R M 1$ identified 3 somatic mutations in the VHL series (13.0\%), whereas 6 cases $(27.3 \%)$ were observed in the sporadic series $(\mathrm{P}=0.28)$. Thus, a total of $9 \mathrm{PBRM1}$ somatic mutations were identified in both series $(20 \%)$ (Fig. 1A, Tables I and SI). To further define the mutational status of $P B R M 1$, we used data from $\mathrm{CGH}$-array hybridization available for some of the tumors. As expected, we observed loss of 3p21 (where PBRM1 is located) in the majority of the tumors $(\mathrm{N}=20 / 24,80 \%)$ and, in particular, in those harboring a PBRMI somatic mutation leading to bi-allelic inactivation of the gene (Fig. 1B and Table SI).

We then used immunohistochemistry (IHC) to determine the level of expression of the PBRM1 protein. The results revealed positive staining that ranged from 0 to $100 \%$. PBRM1-positive staining corresponded to expression of the protein, and a lack of staining indicated an absence of the protein due to a mutation. Overall, we observed that 14 tumors were PBRM1-negative, and 31 tumors were PBRM1-positive. We observed that PBRM1-negative tumors were statistically less frequent in the VHL series $(17.4 \%$, $\mathrm{n}=4)$ compared to the sporadic series $(45.5 \%, \mathrm{n}=10)(\mathrm{P}=0.04)$ (Table I). Most of the tumors exhibited diffuse and high expression of PBRM1 (in two-thirds of the cases more than $70 \%$ of the cells were positive). However, a number of the tumors in both series exhibited a substantial degree of intratumor PBRM1 staining heterogeneity. The VHL-associated RCC samples were less heterogeneous than the sporadic samples (Fig. 2A-D).

A significant association between the results from the PBRM1 sequencing and the PBRM1 IHC was observed. The median percentage of positive cells by IHC in the wild-type PBRM1 tumors (100.0 [85.0-100.0]) was higher than in the mutated PBRM1 tumors (50.0 [30.0-50.0] $(\mathrm{P}=0.0001)$ (Table SII and Fig. SI). The percentage of PBRM1-positive cells in the wild-type PBRM1 tumors $(83.3 \%, \mathrm{n}=30)$ was significantly higher than that in mutated PBRM1 tumors $(11.1 \%, \mathrm{n}=1)(\mathrm{P}=0.0001)$. However, discordance was observed for 6 wild-type $P B R M 1$ cases that lacked PBRM1 expression $(13.3 \%, 6 / 45)$ (Table SII) and one mutated PBRMI case with persistence of nuclear staining. Given the significant association between PBRM1 sequencing and PBRM1 IHC, for a cost-effective strategy, we used the results from the PBRM1 immunostaining to categorize PBRM1-positive vs. PBRM1-negative tumors for the remainder of the analyses. 
Table I. Clinico-pathological features in VHL-associated and sporadic ccRCC.

\begin{tabular}{|c|c|c|c|}
\hline Characteristics & VHL-ccRCC (n=23) & Sporadic ccRCC $(\mathrm{n}=22)$ & P-value \\
\hline Age at diagnosis, years, median (interquartile range) & $40(31-45)$ & $69(59-76)$ & $<0.01$ \\
\hline Sex, \% (n) & & & 0.30 \\
\hline Female & $30.4(7)$ & $45.4(10)$ & \\
\hline Male & $69.6(16)$ & $54.6(12)$ & \\
\hline ISUP grade, $\%(\mathrm{n})$ & & & 0.88 \\
\hline Low, 1-2 & $47.8(11)$ & $50.0(11)$ & \\
\hline High, 3-4 & $52.2(12)$ & $50.0(11)$ & \\
\hline Tumor Stage, \% (n) & & & 0.18 \\
\hline Low, 1-2 & $65.2(15)$ & $45.4(10)$ & \\
\hline High, 3-4 & $34.8(8)$ & $54.6(12)$ & \\
\hline$V H L, \%(\mathrm{n})$ & & & 0.01 \\
\hline Wild-type & $0.0(0)$ & $27.3(6)$ & \\
\hline Mutated & $100.0(23)$ & $72.7(16)$ & \\
\hline HIF subtypes, \% (n) & & & 0.06 \\
\hline Double negative & $0.0(0)$ & $4.6(1)$ & \\
\hline $\mathrm{HIF}^{+}$ & $52.2(12)$ & $40.9(9)$ & \\
\hline $\mathrm{HIF}^{+} / \mathrm{HIF}^{+}$ & $34.8(8)$ & $13.6(3)$ & \\
\hline $\mathrm{HIF}^{+}$ & $13.0(3)$ & $40.9(9)$ & \\
\hline$P B R M 1, \%(\mathrm{n})$ & & & 0.28 \\
\hline Wild-type & $87.0(20)$ & $72.7(16)$ & \\
\hline Mutated & $13.0(3)$ & $27.3(6)$ & \\
\hline PBRM1, \% (n) & & & 0.04 \\
\hline Negative & $17.4(4)$ & $45.5(10)$ & \\
\hline Positive & $82.6(19)$ & $54.5(12)$ & \\
\hline PBRM1 staining, median (interquartile range) & $100.0(80.0-100.0)$ & $80.0(30.0-100.0)$ & 0.12 \\
\hline
\end{tabular}

PBRM1, polybromo 1; VHL, Von Hippel-Lindau; ccRCC, clear-cell renal cell carcinoma; ISUP, International Society of Urological Pathology; HIF, hypoxia inducible factor.

Relationship between pathological features and PBRM1 expression. In 45 tumors, we observed an association between a loss of PBRM1 expression and the tumor stage: the PBRM1-negative tumors had a significantly higher tumor stage $(71.4 \%, \mathrm{n}=10 / 14)$ compared to the PBRM1-positive tumors $(32.3 \%, n=10 / 31)$ $(\mathrm{P}=0.01)$. This association remained significant after controlling for the series $(\mathrm{P}=0.03)$. This association was significant in the sporadic ccRCC $(\mathrm{P}=0.04)$ but not in the VHL tumors $(\mathrm{P}=0.59)$ (Tables II and SIII). There was no association between the PBRM1 expression and the ISUP grade: the PBRM1-negative tumors did not have a significantly higher nucleolar grade $(57.1 \%$, $\mathrm{n}=8 / 14)$ compared to the PBRM1-positive tumors $(48.4 \%$, $\mathrm{n}=15 / 31)(\mathrm{P}=0.59)$, which remained non-significant when controlling for the series (VHL or sporadic tumors) $(\mathrm{P}=0.55)$.

Association between PBRMI and HIF staining. Irrespective of the PBRM1 expression, most of the VHL-ccRCC tumors were either $\mathrm{HIF}^{+}(52.2 \%, \mathrm{n}=12)$ or $\mathrm{HIF}^{+} / \mathrm{HIF}^{+}(34.8 \%, \mathrm{n}=8)$, unlike the sporadic ccRCC tumors, which more often only exhibited HIF2+ staining $(40.9 \%, \mathrm{n}=9)(\mathrm{P}=0.06)$ (Fig. 3A-D, Tables III and SIV). One sporadic tumor was classified as an HIF1-/HIF2-double negative.

We then evaluated the putative relationship between PBRM1 and HIF. Overall, the percentage of HIF1+ or $\mathrm{HIF}^{+} / \mathrm{HIF}^{+}$tumors was $80.6 \%$ in the PBRM1-positive tumors compared to $50.0 \%$ in PBRM1-negative tumors (Table III). However, the association was not significant $(\mathrm{P}=0.13)$. After regrouping the $\mathrm{HIF} 1^{+}$and the $\mathrm{HIF} 1^{+} / \mathrm{HIF}^{+}$ tumors, the association is marginally significant $(\mathrm{P}=0.06)$, whereas no significant association exists when regrouping $\mathrm{HIF}_{2}{ }^{+}$and $\mathrm{HIF}^{+} / \mathrm{HIF}_{2}{ }^{+}(\mathrm{P}=0.29)$.

In the VHL series, the test comparing the distribution of HIFs subtypes between PBRM1-negative and PBRM1-positive tumors was marginally significant $(\mathrm{P}=0.06)$, with a high percentage of $\mathrm{HIF} 1^{+} / \mathrm{HIF} 2^{+}$and a low percentage of $\mathrm{HIF}^{+}$in the PBRM1-positive compared to the PBRM1-negative tumors. In the sporadic ccRCC, no predominant subtype was observed in the distribution of the four subtypes of HIFs $(\mathrm{P}=0.71)$. Similar results were observed in both series after regrouping the $\mathrm{HIF}^{+}$and the $\mathrm{HIF}^{+} / \mathrm{HIF}^{+}{ }^{+}$tumors. In the VHL-ccRCC, $94.7 \% \mathrm{HIF}^{+}{ }^{+}$or $\mathrm{HIF}^{+} / \mathrm{HIF}^{+}{ }^{+}$in the PBRM1-positive tumors were observed compared to $50.0 \%$ in the PBRM1-negative tumors $(\mathrm{P}=0.07)$ and in the sporadic-ccRCC, $58.3 \% \mathrm{HIF}^{+}$or $\mathrm{HIF}^{+} / \mathrm{HIF}^{+}$ were observed in the PBRM1-positive tumors vs. $50.0 \%$ in the PBRM1-negative tumors $(\mathrm{P}=0.82)$. However, there was no significant association after regrouping $\mathrm{HIF}^{+}$with $\mathrm{HIF} 1^{+} / \mathrm{HIF} 2^{+}$in the VHL cohort $(\mathrm{P}=1.00)$. 
A
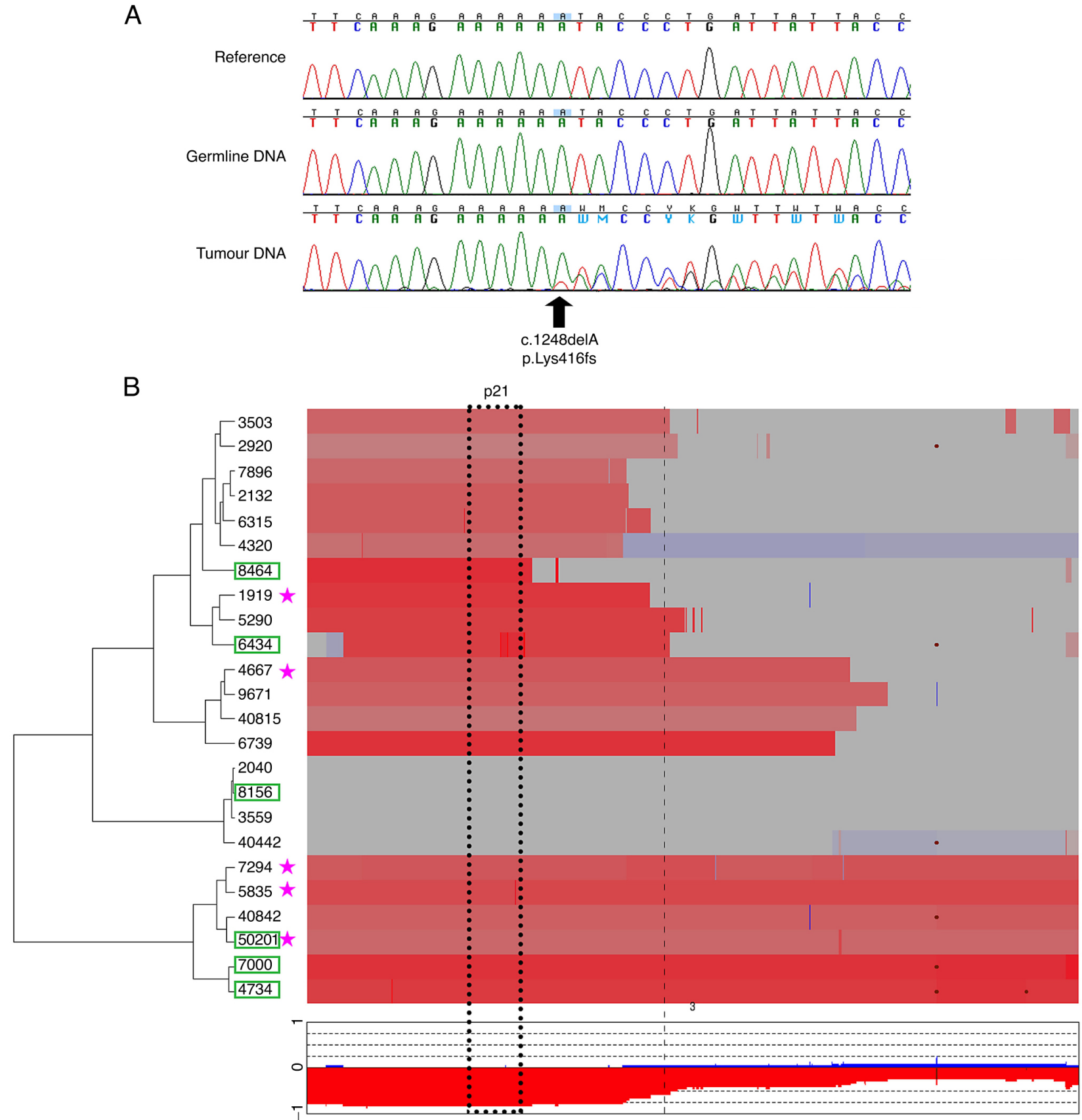

Figure 1. Determination of the mutational status of the $P B R M 1$ gene by sequencing and comparative genomic hybridization-array. (A) Chromatograms showing sequencing of exon 11 for a Von Hippel-Lindau-associated tumor (no. 50201): The frameshift mutation is present only at the somatic level. (B) Clustered heatmap focusing on chromosome 3 showing loss of genomic content for some tumors. Red represents losses, blue represents gains and deletions are shown as brown dots. Bold dashed line represents centromere position, dashed rectangle represents the boundaries of 3p21.x cytobands. Lower panel displays frequency of losses and gains along the chromosome among the tumor series. PBRM1, polybromo 1.

\section{Discussion}

Von Hippel-Lindau disease is the main cause of hereditary renal cell carcinomas, due to germline mutation of the $V H L$ gene, which is the most commonly mutated gene in sporadic ccRCC. The mutation of $V H L$ acts as an initial driver event in tumor development. However, $V H L$ inactivation is not sufficient to initiate tumor formation, thus indicating that additional genetic and/or epigenetic events are involved $(10,11)$. Recent advances in DNA-sequencing techniques have shown that genes coding for proteins involved in the regulation of chromatin remodeling and histone methylation are frequently mutated and closely linked with tumor progression $(14,27)$. Of these genes, PBRM1 is the second most common gene implicated in this process, with somatic mutations occurring in 20 to $40 \%$ of sporadic ccRCC $(12,28)$.

To our knowledge, there have been no reports to date in the literature regarding the involvement of PBRMI somatic mutations and its putative relationship with HIFs specifically in VHL-associated ccRCC. The primary objective of our study was to evaluate the mutational status of PBRM1 in these hereditary tumors compared to sporadic ccRCC tumors. As expected, the clinico-pathological data for both series were concordant with the literature. First, 
Table II. Association between PBRM1 expression by immunohistochemistry and pathological data (tumor stage and ISUP grade) according to VHL-associated and sporadic ccRCC series.

A, Tumor stage

\begin{tabular}{|c|c|c|c|c|c|c|c|c|c|}
\hline \multirow[b]{2}{*}{ PBRM1 expression } & \multicolumn{3}{|c|}{ VHL-ccRCC, $\%$ (n) } & \multicolumn{3}{|c|}{ Sporadic ccRCC, \% (n) } & \multicolumn{3}{|c|}{ Overall, \% (n) } \\
\hline & Low & High & P-value & Low & High & P-value & Low & High & P-value \\
\hline PBRM1 negative & $50.0(2)$ & $50.0(2)$ & $\mathrm{P}=0.59$ & $20.0(2)$ & $80.0(8)$ & $\mathrm{P}=0.04$ & $28.6(4)$ & $71.4(10)$ & $\mathrm{P}=0.01$ \\
\hline PBRM1 positive & $68.4(13)$ & $31.6(6)$ & & $66.7(8)$ & $33.3(4)$ & & $67.7(21)$ & $32.3(10)$ & \\
\hline
\end{tabular}

B, ISUP grade

\begin{tabular}{|c|c|c|c|c|c|c|c|c|c|}
\hline \multirow[b]{2}{*}{ PBRM1 expression } & \multicolumn{3}{|c|}{ VHL-ccRCC, \% (n) } & \multicolumn{3}{|c|}{ Sporadic ccRCC, \% (n) } & \multicolumn{3}{|c|}{ Overall, \% (n) } \\
\hline & Low & High & P-value & Low & High & P-value & Low & High & P-value \\
\hline PBRM1 negative & $25.0(1)$ & $75.0(3)$ & $\mathrm{P}=0.59$ & $50.0(5)$ & $50.0(5)$ & $\mathrm{P}=1.00$ & $42.9(6)$ & $57.1(8)$ & $\mathrm{P}=0.59$ \\
\hline PBRM1 positive & $52.6(10)$ & $47.4(9)$ & & $50.0(6)$ & $50.0(6)$ & & $51.6(16)$ & $48.4(15)$ & \\
\hline
\end{tabular}

P-values were obtained by comparing PBRM1 negative results with PBRM1 positive results. $\mathrm{P}=0.03$ was obtained after controlling for series (VHL and sporadic ccRCC; Cochran-Mantel-Haenszel) in the tumor grade assessment. $\mathrm{P}=0.55$ was obtained after controlling for series (VHL and sporadic ccRCC; Cochran-Mantel-Haenszel) in the ISUP grade assessment. PBRM1, polybromo 1; VHL, Von Hippel-Lindau; ccRCC, clear-cell renal cell carcinoma; ISUP, International Society of Urological Pathology.

A

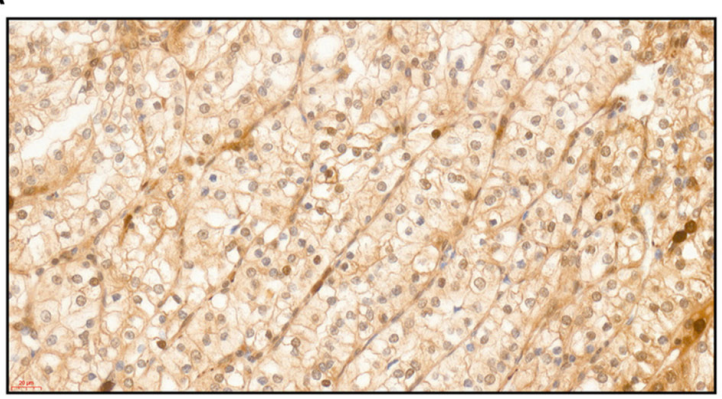

C

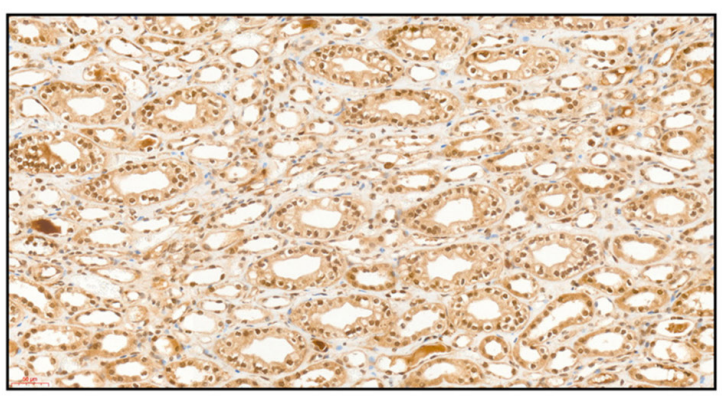

B

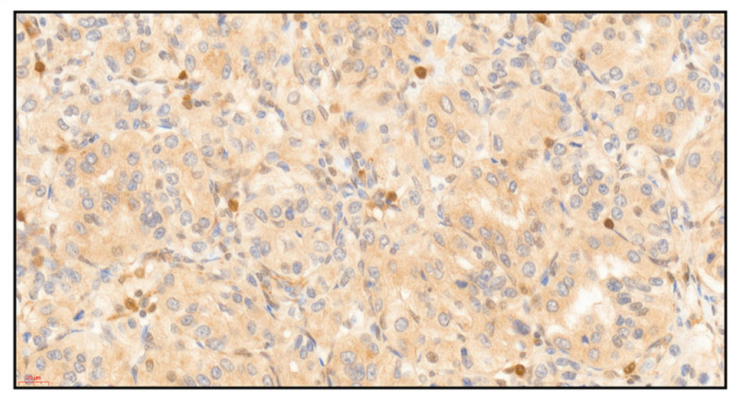

D

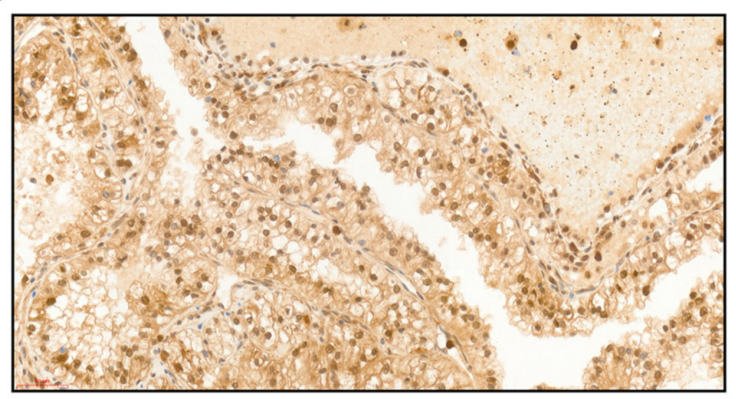

Figure 2. Representative photomicrographs of PBRM1 staining by immunohistochemistry. (A) Heterogeneous nuclear staining of tumor cells in a sporadic ccRCC case (Gx26.8). Scale bar, $20 \mu \mathrm{m}$. (B) PBRM1-negative tumor. All tumor nuclei were uniformly unstained for PBRM1, whereas lymphocytes and stromal cells served as positive controls (Gx26.8). Scale bar, $20 \mu \mathrm{m}$. (C) Non-tumor kidney showing a diffuse nuclear staining of tubular epithelial cells (Gx26.8). Scale bar, $50 \mu \mathrm{m}$. (D) PBRM1-positive tumor. All tumor nuclei were uniformly and strongly stained for PBRM1 in a Von Hippel-Lindau ccRCC with a cystic architecture (Gx26.8). Scale bar, $50 \mu \mathrm{m}$. PBRM1, polybromo 1; ccRCC, clear-cell renal cell carcinoma.

we observed a younger age at diagnosis for patients with VHL-associated tumors compared to those with sporadic ccRCC tumors. Secondly, as expected, there was a high level of $V H L$ somatic mutations $(72.7 \%)$ in the sporadic tumors. We showed that PBRMI somatic mutations can also occur in
VHL-associated ccRCC, at a lower frequency (13.0\%) than in sporadic tumors $(27.3 \%)$ even though this difference is not significant $(\mathrm{P}=0.28)$. Secondly, we analyzed PBRM1 expression by IHC in all cases. We observed significantly higher PBRM1-positive staining in the VHL-ccRCC tumors $(82.6 \%)$ 
Table III. Distribution of HIF subtypes according to PBRM1 expression in VHL-associated and sporadic ccRCC series.

A, VHL-ccRCC

\begin{tabular}{|c|c|c|c|c|c|c|c|}
\hline \multirow[b]{3}{*}{ PBRM1 expression } & \multirow{2}{*}{\multicolumn{4}{|c|}{ HIF subtypes }} & \multicolumn{3}{|c|}{ P-values } \\
\hline & & & & & \multirow{2}{*}{$\begin{array}{l}\text { PBRM1 vs. } \\
\text { PBRM1 vs. all } \\
\text { HIF staining }\end{array}$} & \multirow{2}{*}{$\begin{array}{l}\text { PBRM1 vs. } \\
\mathrm{HIF}^{+} \text {and } \\
\mathrm{HIF}^{+} / \mathrm{HIF}^{+}\end{array}$} & \multirow[b]{2}{*}{$\begin{array}{c}\mathrm{HIF}^{+} \text {and } \\
\mathrm{HIF}^{+} / \mathrm{HIF}^{+}\end{array}$} \\
\hline & $\begin{array}{c}\text { Double Negative, } \\
\%(\mathrm{n})\end{array}$ & $\begin{array}{l}\mathrm{HIF} 1^{+}, \\
\%(\mathrm{n})\end{array}$ & $\begin{array}{c}\mathrm{HIF} 1^{+} / \mathrm{HIF} 2^{+}, \\
\%(\mathrm{n})\end{array}$ & $\begin{array}{l}\mathrm{HIF}^{+} \\
\%(\mathrm{n})\end{array}$ & & & \\
\hline Negative $(n=4)$ & $0.0(0)$ & $50.0(2)$ & $0.0(0)$ & $50.0(2)$ & $\mathrm{P}=0.06$ & $\mathrm{P}=0.07$ & $\mathrm{P}=1.00$ \\
\hline Positive $(n=19)$ & $0.0(0)$ & $52.6(10)$ & $42.1(8)$ & $5.3(1)$ & & & \\
\hline All $(n=23)^{a}$ & $0.0(0)$ & $52.2(12)$ & $34.8(8)$ & $13.0(3)$ & & & \\
\hline
\end{tabular}

B, Sporadic ccRCC

P-values

HIF subtypes

\begin{tabular}{|c|c|c|c|c|c|c|c|}
\hline \multirow[b]{2}{*}{ PBRM1 expression } & & \multirow{2}{*}{$\begin{array}{l}\text { PBRM1 vs. } \\
\text { PBRM1 vs. all } \\
\text { HIF staining }\end{array}$} & \multirow{2}{*}{$\begin{array}{l}\text { PBRM1 vs. } \\
\mathrm{HIF}^{+} \text {and } \\
\mathrm{HIF}^{+} / \mathrm{HIF}^{+}\end{array}$} & \multirow[b]{2}{*}{$\begin{array}{l}\mathrm{HIF}^{+} \text {and } \\
\mathrm{HIF}^{+} / \mathrm{HIF}^{+}\end{array}$} \\
\hline & $\begin{array}{c}\text { Double Negative, } \\
\%(\mathrm{n})\end{array}$ & $\begin{array}{l}\mathrm{HIF1}^{+}, \\
\%(\mathrm{n})\end{array}$ & $\begin{array}{c}\mathrm{HIF}^{+} / \mathrm{HIF}^{+}, \\
\%(\mathrm{n})\end{array}$ & $\begin{array}{l}\mathrm{HIF}^{+}, \\
\%(\mathrm{n})\end{array}$ & & & \\
\hline Negative $(n=10)$ & $10.0(1)$ & $30.0(3)$ & $20.0(2)$ & $40.0(4)$ & $\mathrm{P}=0.71$ & $\mathrm{P}=0.82$ & $\mathrm{P}=0.51$ \\
\hline Positive (n=12) & $0.0(0)$ & $50.0(6)$ & $8.3(1)$ & $41.7(5)$ & & & \\
\hline All $(\mathrm{n}=22)^{\mathrm{a}}$ & $4.6(1)$ & $40.9(9)$ & $13.6(3)$ & $40.9(9)$ & & & \\
\hline
\end{tabular}

C, Overall
HIF subtypes

\begin{tabular}{|c|c|c|c|c|c|c|c|}
\hline \multirow[b]{2}{*}{ PBRM1 expression } & & \multirow{2}{*}{$\begin{array}{l}\text { PBRM1 vs. } \\
\text { PBRM1 vs. all } \\
\text { HIF staining }\end{array}$} & \multirow{2}{*}{$\begin{array}{l}\text { PBRM1 vs. } \\
\mathrm{HIF}^{+} \text {and } \\
\mathrm{HIF}^{+} / \mathrm{HIF} 2^{+}\end{array}$} & \multirow[b]{2}{*}{$\begin{array}{l}\mathrm{HIF}^{+}{ }^{+} \text {and } \\
\mathrm{HIF}^{+} / \mathrm{HIF} 2^{+}\end{array}$} \\
\hline & $\begin{array}{c}\text { Double Negative, } \\
\%(\mathrm{n})\end{array}$ & $\begin{array}{l}\mathrm{HIF}^{+}, \\
\%(\mathrm{n})\end{array}$ & $\begin{array}{c}\mathrm{HIF}^{+} / \mathrm{HIF} 2^{+}, \\
\%(\mathrm{n})\end{array}$ & $\begin{array}{l}\mathrm{HIF}^{+}, \\
\%(\mathrm{n})\end{array}$ & & & \\
\hline Negative $(n=14)$ & $7.1(1)$ & $35.7(5)$ & $14.3(2)$ & $42.9(6)$ & $\mathrm{P}=0.13$ & $\mathrm{P}=0.06$ & $\mathrm{P}=0.29$ \\
\hline Positive $(\mathrm{n}=31)$ & $0.0(0)$ & $51.6(16)$ & $29.0(9)$ & $19.4(6)$ & & & \\
\hline All $(n=45)$ & $2.0(1)$ & $47.0(21)$ & $24.0(11)$ & $27.0(12)$ & & & \\
\hline
\end{tabular}

P-values were obtained by comparing HIF subtypes with PBRM1 expression. P=0.43 was obtained after controlling for series (VHL and sporadic ccRCC; Cochran-Mantel-Haenszel) in the overall group. When regrouping HIF $1^{+}$with $\mathrm{HIF} 1^{+} / \mathrm{HIF} 2^{+}, \mathrm{P}=0.26$ was obtained, which was not significant. When regrouping $\mathrm{HIF} 2^{+}$with $\mathrm{HIF} 1^{+} / \mathrm{HIF} 2^{+}, \mathrm{P}=0.44$ was obtained, which was not significant. ${ }^{\mathrm{a}} \mathrm{P}-\mathrm{value}$ comparing $\mathrm{HIF}$ distribution between VHL-ccRCC and sporadic ccRCC was 0.06. PBRM1, polybromo 1; VHL, Von Hippel-Lindau; ccRCC, clear-cell renal cell carcinoma; HIF, hypoxia inducible factor. compared to the sporadic tumors $(54.5 \%)(\mathrm{P}=0.04)$. However, there was a degree of discordance between sequencing and IHC results in $15.5 \%$ of our cases concerning 6 wild-type PBRM1 cases that lacked PBRM1 expression. This can be explained by the fact that mutations can be missed (in particular insertions and deletions of entire exons) when Sanger sequencing is used. Moreover, the hypermethylation status was not analyzed. However, we observed by $\mathrm{CGH}$ array a loss of chromosome $3 \mathrm{p}$ as expected in the majority of tumors. Thus, PBRM1 can be inactivated on both alleles in some tumors. For immunohistochemistry, preanalytical steps may explain some of the discrepancies. The discordance between sequencing and IHC results for 7 patients leads to similar association between PBRM1 mutational status and tumor stage for overall series. However, this association was significant in the VHL tumors $(\mathrm{P}=0.03)$ but not in the sporadic ccRCC $(\mathrm{P}=0.16)$.

Interestingly, there was a significant overall association between PBRM1 and the tumor stage $(\mathrm{P}=0.03)$ (a higher stage in the PBRM1-negative compared to the PBRM1-positive tumors) but not for the ISUP grade $(\mathrm{P}=0.55)$ after controlling for the series (VHL and sporadic ccRCC). The significant association between PBRM1 and the tumor stage was also observed in the sporadic ccRCC $(\mathrm{P}=0.04)$ but not in the VHL-ccRCC $(\mathrm{P}=0.59)$, which is probably due to a lack of power and/or a recruitment bias. The former has recently been reported in sporadic ccRCC (29). This difference between the VHL and the sporadic series can be explained 
A

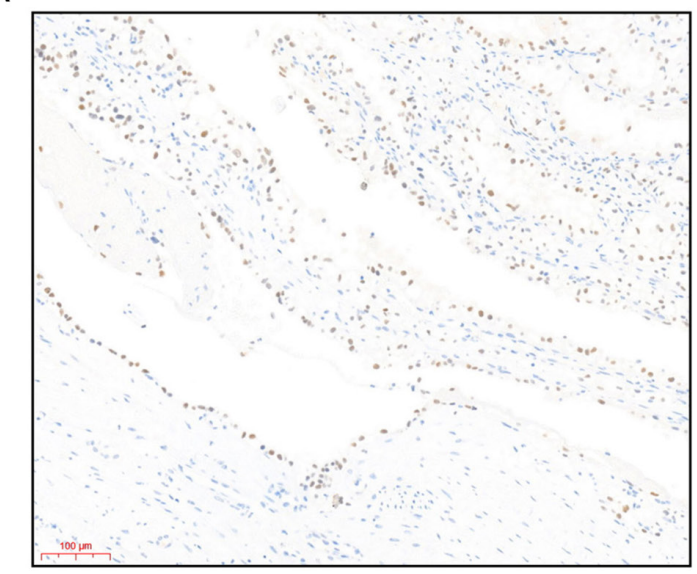

C

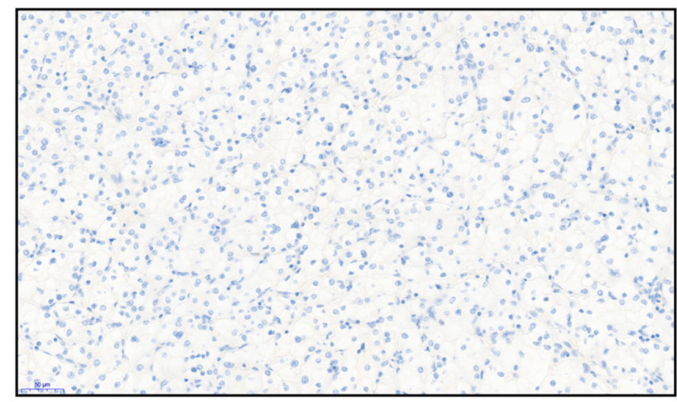

B

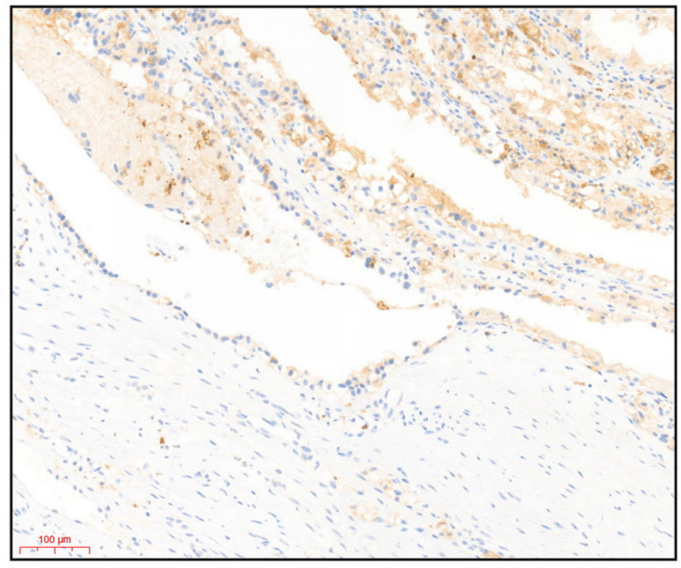

D

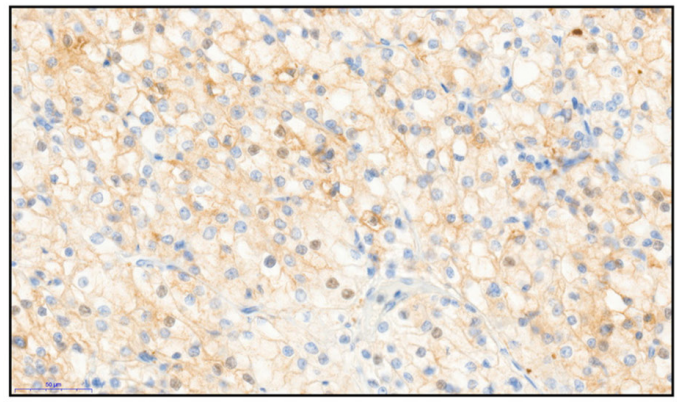

Figure 3. Representative photomicrographs of HIF staining by immunohistochemistry. (A) VHL tumor, diffuse expression of HIF1 in tumor nuclei (Gx20.2). Scale bar, $100 \mu \mathrm{m}$. (B) VHL tumor, negative expression of HIF2 (Gx20.2). Scale bar, $100 \mu \mathrm{m}$. (C) Sporadic tumor, negative HIF1 expression (Gx29). Scale bar, $50 \mu \mathrm{m}$. (D) Sporadic tumor, positive nuclear HIF2 expression in dispersed tumor cells (Gx29). Scale bar, $50 \mu \mathrm{m}$. HIF, hypoxia inducible factor; VHL, Von Hippel-Lindau.

by the fact that VHL-ccRCC are generally diagnosed earlier than sporadic tumors and they most often have a low stage and grade $(3,30)$. The result regarding association between PBRM1 and the tumor stage would suggest that loss of PBRM1 could be a later event in VHL-associated renal carcinogenesis.

As contradictory results regarding the relationship between PBRM1 and HIFs have been reported, the secondary objective of our study was to investigate this putative link in our own series $(19,20)$. We stratified the HIF immunostaining for our series into four categories: $\mathrm{HIF}^{+}$, HIF2 $2^{+}$, $\mathrm{HIF}^{+} / \mathrm{HIF}^{+}$, and double-negative tumors. We observed a marginal association between both series and the distribution of the HIF subtypes $(\mathrm{P}=0.06)$ : more $\mathrm{HIF} 1^{+}$tumors were observed in VHL-associated ccRCC (52.2\%) than in sporadic ccRCC (40.9\%). As these hereditary tumors are generally less aggressive and they consequently have a better prognosis $(3,30)$, this result would suggest that HIF1 expression could be less oncogenic than HIF2 expression, as already reported (5-9). When investigating the association between PBRM1 mutation status and HIF, no significant association was observed but these results are based on only 3 mutated patients in VHL cohort.

Furthermore, in our VHL-associated series, the PBRM1-positive tumors (wild-type PBRMI) were more frequently classified as $\mathrm{HIF} 1^{+}$or $\mathrm{HIF} 1^{+} / \mathrm{HIF} 2^{+}$. This is in accordance with reports that HIF1 is probably acting as a tumor suppressor, and the loss or mutation of PBRMI being a later event $(6,15,19)$. Two major studies have focused on the relationship between PBRM1 and HIFs in human cell lines and in tissue samples. The first study, conducted by Gao et al, has shown that inactivation of PBRM1 amplifies the HIF response in cell lines (19). In our study, HIF expression was observed in both the PBRM1-positive and the PBRM1-negative tumors, without an increase in the number of cases with HIF-positive staining when tumors were PBRM1 negative. In the second study, Murakami et al showed in cell lines and in tumor samples that PBRM1 interacts differently according to the expression of $\mathrm{HIF} 1^{+} / \mathrm{HIF}^{+}$or $\mathrm{HIF}^{+}{ }^{+}(20)$. The authors described, on the one hand, that loss of PBRM1 was observed only in the $\mathrm{HIF}^{+} / \mathrm{HIF}^{+}$cell lines, thus leading to reduced HIF1 tumor-suppressive activity. On the other hand, they observed that the HIF2+ subtype was associated with PBRM1 expression. In our series, most of the cases expressing only HIF $1^{+}$were PBRM1-positive, but we did not observe a significant association between the PBRM1 status and HIF after regrouping $\mathrm{HIF}^{+}{ }^{+}$with $\mathrm{HIF}^{+} / \mathrm{HIF}^{+}(\mathrm{P}=0.26)$. However, in these two studies the methodologies were different from ours: use of tumor cell lines or sporadic ccRCC, but not VHL-associated ccRCC, immunohistochemistry performed on tissue microarray and not on whole slide, analysis of few tumor specimens, use of different commercial antibodies, and analysis of only HIF1 and not HIF2 expression. This could explain the discrepancy with our results. Finally, Nargund et al reported a mouse model for which, after the loss of $V h l$, the loss of Pbrml further activated the HIF1 pathway (31). Increasing the number of VHL-associated and sporadic ccRCC, with 
additional experiments, could lead to a better understanding of the relationship between HIF factors and PBRM1 in renal carcinogenesis with adequate statistical power to detect any statistical significant associations through the analyses of stratified data. It is also possible that other components interact with PBRM1 and/or HIFs.

It has been widely observed that sporadic ccRCC are morphologically heterogeneous (32). Mutation profiles obtained by next-generation sequencing have recently confirmed intratumor heterogeneity in ccRCC at the molecular level, in particular in sporadic cases $(33,34)$. In our study, we observed intratumor heterogeneity in terms of PBRM1 staining, with the VHL-associated cases being less heterogeneous than the sporadic ccRCC. It would be interesting to further study several synchronous tumors arising in VHL patients, in particular for PBRM1 staining, and sub-classification of the HIF factors (24).

In conclusion, we have shown that in VHL-associated ccRCC, somatic mutations in the PBRMI gene can occur and that PBRM1-positive staining is significantly higher compared to the sporadic tumors. Secondly, PBRM1 expression is more likely to be associated with HIF1 than with HIF2 expression in VHL cohort. Our results would suggest that co-expression of PBRM1 and HIF1 would have a less oncogenic role in the VHL-associated ccRCC.

\section{Acknowledgements}

The authors would like to thank the 'Biological Resource Center' from Necker Hospital (Paris, France), Bicêtre Hospital (Le Kremlin-Bicêtre, France) and St Joseph Hospital (Paris, France) for providing the frozen and fixed tumor samples.

\section{Funding}

This work was supported by grants from the 'French League Against Cancer' (Cher and Indre committees), the French National Cancer Institute (INCa, PNES Kidney cancer), the 'Association VHL France' and by 'Tax learning Gustave Roussy' (grant no. P18_SG_CGH).

\section{Availability of data and materials}

The datasets used and/or analyzed during the current study are available from the corresponding author on reasonable request.

\section{Authors' contributions}

SG, SF and SR developed the study design, collected samples and data, interpreted data and drafted the manuscript. GLT performed statistical analysis, interpreted data and drafted the manuscript. BN, BGr, MDC and ND conceived and carried out genetic experiments, and participated in drafting the manuscript. KP carried out immunohistochemical experiments. VV, VD VM and AM collected samples and data, and participated in drafting the manuscript. BJ and GM analyzed bioinformatics data and participated in drafting the manuscript. SC and FR helped to design the study, to prepare the figures, and participated in drafting the manuscript. BGa and BTT helped to design the study and interpret data, and critically revised the manuscript. All authors read and approved the final manuscript. SG and SF confirm the authenticity of all the raw data.

\section{Ethics approval and consent to participate}

The present study was approved by the CCPRB (French Ethical Committee) Paris-Sud at Bicêtre Hospital (Le Kremlin-Bicêtre, France): Genetic study of von Hippel-Lindau disease (approval no. 01-2001). All patients provided written informed consent prior to surgery for the use of their tumors.

\section{Patient consent for publication}

Not applicable.

\section{Competing interests}

The authors declare that they have no competing interests.

\section{Authors' information}

The ORCID IDs for the authors of this study are as follows: SG, 0000-0002-3158-6791 and SF, 0000-0003-4124-9227.

\section{References}

1. Scelo G and Larose TL: Epidemiology and risk factors for kidney cancer. J Clin Oncol 36: JCO2018791905, 2018.

2. Moch H, Cubilla AL, Humphrey PA, Reuter VE and Ulbright TM: The 2016 WHO classification of tumours of the urinary system and male genital organs-part A: Renal, penile, and testicular tumours. Eur Urol 70: 93-105, 2016.

3. Richard S, Gardie B, Couvé S and Gad S: Von Hippel-Lindau: How a rare disease illuminates cancer biology. Semin Cancer Biol 23: 26-37, 2013.

4. Shen $C$ and Kaelin WG Jr: The VHL/HIF axis in clear cell renal carcinoma. Semin Cancer Biol 23: 18-25, 2013.

5. Gordan JD, Lal P, Dondeti VR, Letrero R, Parekh KN, Oquendo CE, Greenberg RA, Flaherty KT, Rathmell WK, Keith $\mathrm{B}$, et al: HIF-alpha effects on c-Myc distinguish two subtypes of sporadic VHL-deficient clear cell renal carcinoma. Cancer Cell 14: 435-446, 2008.

6. Meléndez-Rodríguez F, Roche $\mathrm{O}$, Sanchez-Prieto $\mathrm{R}$ and Aragones J: Hypoxia-inducible factor 2-dependent pathways driving von Hippel-Lindau-deficient renal cancer. Front Oncol 8: $214,2018$.

7. Hoefflin R, Harlander S, Schäfer S, Metzger P, Kuo F, Schönenberger D, Adlesic M, Peighambari A, Seidel P, Chen CY, et al: HIF- $1 \alpha$ and HIF- $2 \alpha$ differently regulate tumour development and inflammation of clear cell renal cell carcinoma in mice. Nat Commun 11: 4111, 2020.

8. Choueiri TK and Kaelin WG Jr: Targeting the HIF2-VEGF axis in renal cell carcinoma. Nat Med 26: 1519-1530, 2020.

9. Semenza GL: Heritable disorders of oxygen sensing. Am J Med Genet A 185: 2576-2581, 2021.

10. Kim WY and Kaelin WG: Role of VHL gene mutation in human cancer. J Clin Oncol 22: 4991-5004, 2004.

11. Mandriota SJ, Turner KJ, Davies DR, Murray PG, Morgan NV, Sowter HM, Wykoff CC, Maher ER, Harris AL, Ratcliffe PJ and Maxwell PH: HIF activation identifies early lesions in VHL kidneys: Evidence for site-specific tumor suppressor function in the nephron. Cancer Cell 1: 459-468, 2002.

12. Varela I, Tarpey P, Raine K, Huang D, Ong CK, Stephens P, Davies H, Jones D, Lin ML, Teague J, et al: Exome sequencing identifies frequent mutation of the SWI/SNF complex gene PBRM1 in renal carcinoma. Nature 469: 539-542, 2011.

13. Peña-Llopis S, Vega-Rubín-de-Celis S, Liao A, Leng $\mathrm{N}$, Pavía-Jiménez A, Wang S, Yamasaki T, Zhrebker L, Sivanand S, Spence P, et al: BAP1 loss defines a new class of renal cell carcinoma. Nat Genet 44: 751-759, 2012. 
14. Dalgliesh GL, Furge K, Greenman C, Chen L, Bignell G, Butler A, Davies H, Edkins S, Hardy C, Latimer C, et al: Systematic sequencing of renal carcinoma reveals inactivation of histone modifying genes. Nature 463: 360-363, 2010.

15. Hsieh JJ, Le VH, Oyama T, Ricketts CJ, Ho TH and Cheng EH: Chromosome $3 p$ loss-orchestrated VHL, HIF, and epigenetic deregulation in clear cell renal cell carcinoma. J Clin Oncol 36: JCO2018792549, 2018.

16. Benusiglio PR, Couvé S, Gilbert-Dussardier B, Deveaux S, Le Jeune H, Da Costa M, Fromont G, Memeteau F, Yacoub M, Coupier I, et al: A germline mutation in PBRM1 predisposes to renal cell carcinoma. J Med Genet 52: 426-430, 2015.

17. Chowdhury B, Porter EG, Stewart JC, Ferreira CR, Schipma MJ and Dykhuizen EC: PBRM1 regulates the expression of genes involved in metabolism and cell adhesion in renal clear cell carcinoma. PLoS One 11: e0153718, 2016.

18. Hargreaves DC and Crabtree GR: ATP-dependent chromatin remodeling: Genetics, genomics and mechanisms. Cell Res 21: 396-420, 2011.

19. Gao W, Li W, Xiao T, Liu XS and Kaelin WG Jr: Inactivation of the PBRM1 tumor suppressor gene amplifies the HIF-response in $\mathrm{VHL}^{-1-}$ clear cell renal carcinoma. Proc Natl Acad Sci USA 114: 1027-1032, 2017

20. Murakami A, Wang L, Kalhorn S, Schraml P, Rathmell WK, Tan AC, Nemenoff R, Stenmark K, Jiang BH, Reyland ME, et al: Context-dependent role for chromatin remodeling component PBRM1/BAF180 in clear cell renal cell carcinoma. Oncogenesis 6: e287, 2017.

21. Beroukhim R, Brunet JP, Di Napoli A, Mertz KD, Seeley A Pires MM, Linhart D, Worrell RA, Moch H, Rubin MA, et al: Patterns of gene expression and copy-number alterations in von-hippel lindau disease-associated and sporadic clear cell carcinoma of the kidney. Cancer Res 69: 4674-4681, 2009.

22. Mitchell TJ, Turajlic S, Rowan A, Nicol D, Farmery JHR, O'Brien T, Martincorena I, Tarpey P, Angelopoulos N, Yates LR, et al: Timing the landmark events in the evolution of clear cell renal cell cancer: TRACERx renal. Cell 173: 611-623. e17, 2018.

23. Delahunt B, Cheville JC, Martignoni G, Humphrey PA, Magi-Galluzzi C, McKenney J, Egevad L, Algaba F, Moch H, Grignon DJ, et al: The International society of urological pathology (ISUP) grading system for renal cell carcinoma and other prognostic parameters. Am J Surg Pathol 37: 1490-1504, 2013.

24. Gattolliat CH, Couvé S, Meurice G, Oréar C, Droin N, Chiquet M, Ferlicot S, Verkarre V, Vasiliu V, Molinié V, et al: Integrative analysis of dysregulated microRNAs and mRNAs in multiple recurrent synchronized renal tumors from patients with von Hippel-Lindau disease. Int J Oncol 53: 1455-1468, 2018

25. Messai Y, Gad S, Noman MZ, Le Teuff G, Couve S, Janji B, Kammerer SF, Rioux-Leclerc N, Hasmim M, Ferlicot S, et al: Renal cell carcinoma programmed death-ligand 1, a new direct target of hypoxia-inducible factor- 2 alpha, is regulated by von hippel-lindau gene mutation status. Eur Urol 70: 623-632, 2016.
26. Nam SJ, Lee C, Park JH and Moon KC: Decreased PBRM1 expression predicts unfavorable prognosis in patients with clear cell renal cell carcinoma. Urol Oncol 33: 340.e9-16, 2015.

27. Jiang W, Dulaimi E, Devarajan K, Parsons T, Wang Q, Liao L, Cho EA, O'Neill R, Solomides C, Peiper SC, et al: Immunohistochemistry successfully uncovers Intratumoral heterogeneity and widespread co-losses of chromatin regulators in clear cell renal cell carcinoma. PLoS One 11: e0164554, 2016.

28. Gossage L, Murtaza M, Slatter AF, Lichtenstein CP, Warren A, Haynes B, Marass F, Roberts I, Shanahan SJ, Claas A, et al: Clinical and pathological impact of VHL, PBRM1, BAP1, SETD2, KDM6A, and JARID1c in clear cell renal cell carcinoma. Genes Chromosomes Cancer 53: 38-51, 2014

29. Bihr S, Ohashi R, Moore AL, Rüschoff JH, Beisel C, Hermanns T, Mischo A, Corrò C, Beyer J, Beerenwinkel N, et al: Expression and mutation patterns of PBRM1, BAP1 and SETD2 mirror specific evolutionary subtypes in clear cell renal cell carcinoma. Neoplasia 21: 247-256, 2019.

30. Neumann HP, Bender BU, Berger DP, Laubenberger J, SchultzeSeemann W, Wetterauer U, Ferstl FJ, Herbst EW, Schwarzkopf G Hes FJ, et al: Prevalence, morphology and biology of renal cell carcinoma in von Hippel-Lindau disease compared to sporadic renal cell carcinoma. J Urol 160: 1248-1254, 1998.

31. Nargund AM, Pham CG, Dong Y, Wang PI, Osmangeyoglu HU, Xie Y, Aras O, Han S, Oyama T, Takeda S, et al: The SWI/SNF protein PBRM1 restrains VHL-loss-driven clear cell renal cell carcinoma. Cell Rep 18: 2893-2906, 2017.

32. Delahunt B, Grignon DJ and Eble JN: Tumors of the kidney. In: Urological Pathology. Lippincott Williams \& Wilkins, a Wolters Kluwer business, Philadelphia, PA, USA, pp 72-204, 2014.

33. Gerlinger M, Rowan AJ, Horswell S, Math M, Larkin J, Endesfelder D, Gronroos E, Martinez P, Matthews N, Stewart A, et al: Intratumor heterogeneity and branched evolution revealed by multiregion sequencing. N Engl J Med 366: 883-892, 2012.

34. Fisher R, Horswell S, Rowan A, Salm MP, de Bruin EC, Gulati S, McGranahan N, Stares M, Gerlinger M, Varela I, et al: Development of synchronous VHL syndrome tumors reveals contingencies and constraints to tumor evolution. Genome Biol 15: 433, 2014.

This work is licensed under a Creative Commons

Attribution-NonCommercial-NoDerivatives 4.0 International (CC BY-NC-ND 4.0) License. 